\title{
Caracterización reológica de soluciones azucaradas para el proceso de evaporación-cristalización
}

\author{
A. Martínez Gómez ${ }^{1}$, I. Sánchez Bazán ${ }^{1}$, A. Osorio-Mirón ${ }^{1 *}$, L. López Zamora², \\ L.A. Sánchez Bazán ${ }^{1}$ E. Hernández Aguilar ${ }^{1}$ \\ ${ }^{1}$ Universidad Veracruzana, Facultad de Ciencias Químicas, Prolong. Oriente 6 No. 1009, Col. Rafael Alvarado, \\ C.P. 94340 Orizaba, Veracruz, México \\ E-mail: anosorio@uv.mx \\ ${ }^{2}$ Instituto Tecnológico de Orizaba, DEPI-MCIQ, Oriente 9 No. 852, Col. E. Zapata, \\ C.P. 94320 Orizaba, Veracruz, México
}

(recibido/received: 31-Enero-2011; aceptado/accepted: 17-Junio-2011)

\begin{abstract}
RESUMEN
En este trabajo se realizó un estudio experimental para la caracterización reológica de soluciones azucaradas en el proceso de producción de azúcar. El objetivo principal fué la caracterización de soluciones azucaradas con el fin de modelar su comportamiento reológico para la optimización del proceso de evaporación-cristalización en una etapa posterior. En los experimentos, se utilizó un diseño de experimento tipo factorial $3^{\mathrm{k}}$, en donde se considera la interacción de tres factores en la determinación de la variable de respuesta; en este caso los factores son: la concentración $\left({ }^{\circ} \mathrm{Bx}\right)$, la temperatura y las revoluciones por minuto (velocidad de corte), cada uno con tres niveles, tomando como variable de respuesta la viscosidad. El desarrollo experimental y el análisis de resultados conduce a la determinación del modelo reológico de las soluciones problema en el rango de estudio de los factores de interés, proponiéndose un modelo tipo Ostwald-de Waele para fluidos dilatantes.
\end{abstract}

Palabras claves: Modelo reológico; Velocidad de corte; Viscosidad

\begin{abstract}
In this work, an experimental study for the rheological characterisation of sugar solutions in the process of sugar production was performed. The main purpose was the characterisation of sugar solutions in order to model its rheological behaviour for optimising the evaporation-crystallisation process at a later stage. In the experiments, a $3^{\mathrm{k}}$ factorial design of experiment was used, where it considers the interaction of three factors in determining the response variable, in this case the factors are: the concentration $\left({ }^{\circ} \mathrm{Bx}\right)$, the temperature and revolutions per minute (shear rate), each one with three levels, taking as response variable viscosity. The experimental development and analysis of results lead to the determination of the rheological model for the test solutions in the study range of the factors of interest, and it is proposed a type Ostwald-de Waele model for dilatant fluids.
\end{abstract}

Keywords: Rheological model; Shear rate; Viscosity

*Autor para la correspondencia 


\section{INTRODUCCIÓN}

La caracterización de soluciones en la industria de procesos es de primordial importancia para efectuar acciones y toma de decisiones. En el caso de materias primas y productos, es de gran utilidad para el control de procesos, sin embargo el comportamiento reológico de diversas soluciones es muy complejo y desconocido, y a la vez desempeña un papel muy importante en muchos sistemas de proceso. La producción de azúcar de caña es un proceso altamente complejo debido a las diferentes etapas en las que el jugo de caña es tratado hasta obtener los cristales de sacarosa.

La cristalización es importante como proceso industrial por los diferentes materiales que son comercializados en forma de cristales como es el caso del azúcar o sacarosa. Las propiedades reológicas son importantes en los procesos de fabricación del azúcar, y aun son desconocidas por una gran parte de los técnicos de la industria azucarera, no disponiéndose en la actualidad de datos confiables que permitan establecer su magnitud en cualquiera de las condiciones de proceso.

La caracterización de soluciones azucaradas permite predecir el comportamiento dentro de un cristalizador industrial contribuyendo al agotamiento adecuado de las mismas. Se ha observado que la viscosidad de las soluciones azucaradas cambia en función del crecimiento de los cristales de azúcar, el contenido de impurezas y la cantidad de agua evaporada (Chen y Chen, 2001).

En la evaporación-cristalización, la caracterización de los materiales utilizando, ecuaciones constitutivas, que relacionan los esfuerzos aplicados sobre éstos y las deformaciones que presentan, es de gran relevancia; debido a esto, el objetivo principal de este trabajo es la caracterización de soluciones azucaradas con el fin de modelar su comportamiento reológico para la optimización del proceso de evaporación-cristalización en una etapa posterior.
El trabajo está distribuido como sigue: En la sección siguiente se describe la infraestructura experimental disponible y se presenta el diseño de experimentos a seguir (metodología), se considerán tres factores a evaluar con la viscosidad como variable de respuesta. Enseguida, se presenta la discusión de los resultados experimentales y el análisis numérico estadístico realizado para proponer el modelo reológico que describe el comportamiento de las soluciones azucaradas en el rango de experimentación propuesto. Finalmente se presentan algunas conclusiones del trabajo numérico experimental llevado a cabo.

\section{METODOLOGÍA}

\section{Teoría}

La caracterización de los materiales utilizando ecuaciones constitutivas que relacionan los esfuerzos aplicados sobre éste y las deformaciones que él sufre, es el campo de estudio de la reología. Esta caracterización se basa en la ley de Newton de la viscosidad la cual establece la proporcionalidad existente entre el esfuerzo por unidad de área (F/A) necesario para producir un gradiente de velocidades en un fluido, siendo la constante de proporcionalidad un factor que describe "la capacidad de deslizamiento de un fluido".

Los fluidos no-Newtonianos son aquellos fluidos que no cumplen la ley de Newton de la viscosidad, por lo tanto, la relación entre el esfuerzo cortante y la velocidad de deformación deja de ser lineal. Estos fluidos se caracterizan porque su viscosidad no permanece constante cuando la temperatura y la composición permanecen invariables, sino que depende del esfuerzo cortante o gradiente de velocidad y, a veces, del tiempo de aplicación del esfuerzo y de la historia previa del producto o muestra. Ello se debe a que la constitución física varía al someter el producto a los efectos de rozamiento a lo largo del tiempo. Dado que en este tipo de fluidos la viscosidad no permanece constante, se define a la viscosidad aparente como la relación entre el esfuerzo cortante y la velocidad de deformación correspondiente. Estos fluidos se 
diferencian en dependientes e independientes del tiempo.

Los fluidos independientes del tiempo, se clasifican de acuerdo al esfuerzo umbral, es decir, si necesitan un esfuerzo cortante mínimo para que el fluido se ponga en movimiento.

Los fluidos sin esfuerzo umbral, como los fluidos pseudoplásticos (shear thinning), los cuales se caracterizan por una disminución de su viscosidad, al aplicársele un esfuerzo constante, y si la velocidad es lo suficiente rápida, la viscosidad se estabiliza en un valor pequeño. De este modo, la viscosidad de un fluido depende de la intensidad del esfuerzo de corte o cizallamiento.

Algunos ejemplos de fluidos pseudoplásticos son: tipos de salsa de tomate, mostaza, algunas clases de pinturas, suspensiones acuosas de arcilla, la solución concentrada de azúcar a partir de la caña, entre otras, considerando que se tiene una tendencia logarítmica como se muestra en la Fig. 1.
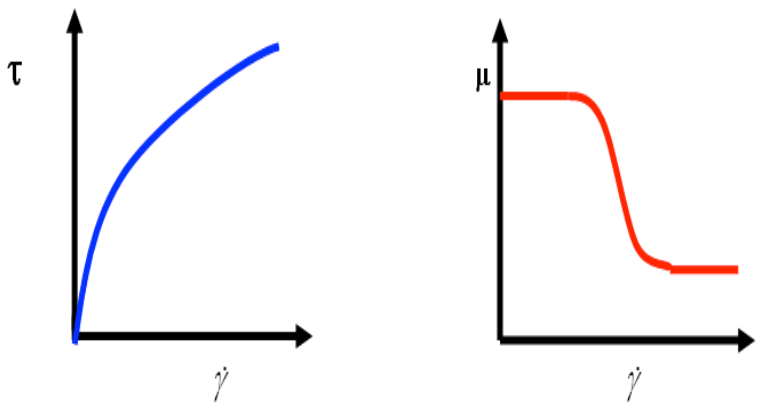

Fig. 1 Curvas de fluidez y de viscosidad para un fluido pseudoplástico (Díaz y Remedios, 2002).

La ecuación reológica de estos fluidos sigue el modelo de Ostwald o Ley de potencia, cuya expresión es la siguiente:

$$
\tau=K\left(\frac{d V}{d t}\right)-K(\hat{\gamma})^{n} \quad 1<\mathrm{n}>0
$$

donde $\tau$ es el esfuerzo cortante, $\hat{\gamma}$ es la velocidad de deformación, $K$ es una constante cuyas dimensiones dependen del valor de $\eta$ (viscosidad aparente) y $n$ es un valor menor que uno. La ordenada en el origen que se obtiene representa el valor de $K$.

\section{Equipos}

Los equipos disponibles para la realización de experimentos que permitan determinar la viscosidad de fluidos en función del esfuerzo de corte para la elaboración de perfiles reologicos (Brookfield, 2003) incluye un viscosímetro RVDVE marca Brookfield acondicionado con un rotavapor A404-2 con rango de temperatura de 0 a $120{ }^{\circ} \mathrm{C}$ y un termómetro a infrarrojo marca "Fluke" con un rango de -30 a $500{ }^{\circ} \mathrm{C}$. Estos equipos se muestran en la Fig. 2.

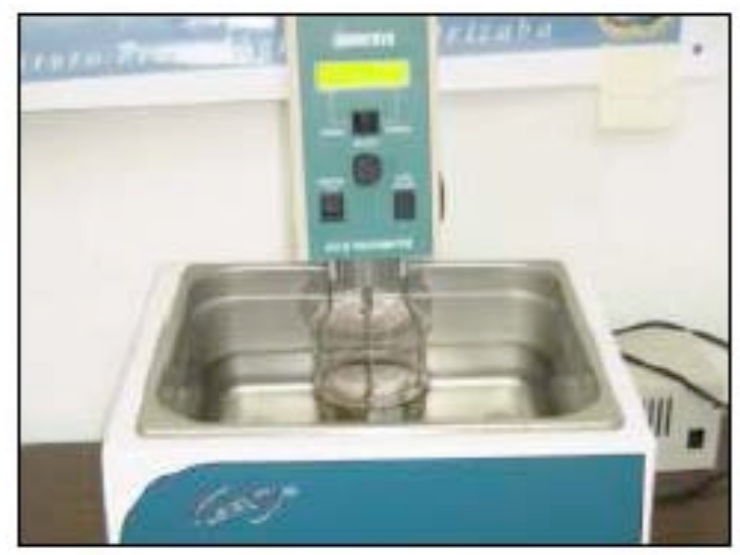

Fig. 2 Viscosímetro Brookfield RV-DVE y Rotavapor A404-2.

Los viscosímetros Brookfield son utilizados frecuentemente para realizar mediciones de viscosidad de una variedad de materiales con comportamiento Newtoniano y no-Newtoniano. Debido a la importancia de tales mediciones en muchos campos de la industria, existe la necesidad de incluir las mediciones con estos equipos en los sistemas de aseguramiento de calidad.

El viscosímetro Brookfield RV-DVE proporciona una medida de la viscosidad de un fluido a determinado esfuerzo de corte, así la viscosidad es una medida de la resistencia de un líquido a fluir (Brookfield, 2003). Todos los viscosímetros Brookfield utilizan el conocido principio de la viscosimetría rotacional; el 
husillo o eje que se sumerge en el fluido en estudio esta acoplado por medio de un resorte calibrado a un motor de velocidad variable. Cuando el husillo o eje gira la deformación del resorte es proporcional al esfuerzo de torsión necesario para vencer la fuerza de resistencia viscosa, indicándose en la pantalla digital el valor de la deformación que es proporcional a la viscosidad del fluido (Shames, 1999).

Cuanto mayor es el esfuerzo de torsión, más alto es el rango de medición. Las unidades de medición se muestran de acuerdo al sistema a utilizar, ya sea cegesimal (cP) o Sistema Internacional (mPa•s); la lectura de la viscosidad aparece en las unidades de centipoise (cP) o miliPascal segundos (mPa•s) y el esfuerzo en unidades dinas-centímetros o Newton-metros (que se muestra como porcentaje "\%") en la pantalla. El equivalente en unidades de medida del sistema SI, se calcularán mediante las conversiones mostradas en la Tabla 1.

Tabla 1 Equivalencia de unidades de viscosidad en el SI y CGS.

\begin{tabular}{ccc}
\hline Variable & $\begin{array}{c}\text { Sistema } \\
\text { Internacional }\end{array}$ & $\begin{array}{c}\text { Sistema } \\
\text { Cegesimal }\end{array}$ \\
\hline $\begin{array}{c}\text { Viscosidad } \\
\text { Esfuerzo de } \\
\text { torsión }\end{array}$ & $1 \mathrm{mPa} \bullet \mathrm{s}$ & $1 \mathrm{cP}$ \\
\hline
\end{tabular}

Un refractómetro de mano marca "ATAGO HRS-500" con un rango de 0-90 ${ }^{\circ} \mathrm{Bx}$, para la medición se divide en tres posiciones: de 0 a 30 ${ }^{\circ} \mathrm{Bx}$ de 31 a $60{ }^{\circ} \mathrm{Bx}$ y de 61 a $90{ }^{\circ} \mathrm{Bx}$, este refractómetro se muestra en la Fig. 3.

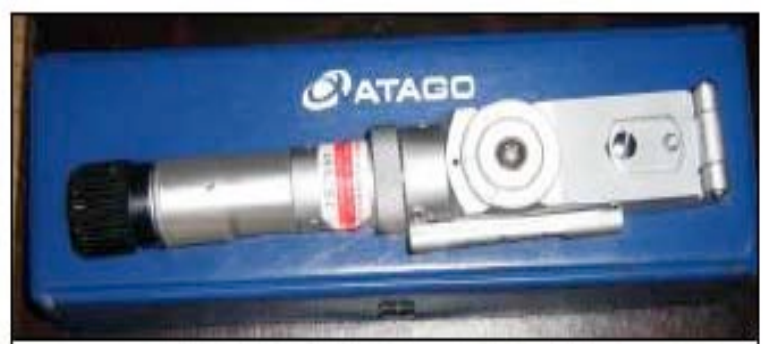

Fig. 3 Refractómetro de mano ATAGO.

\section{Diseño de experimentos}

Se sigue un diseño de experimentos tipo factorial $3^{\mathrm{k}}$ con el que se pretende observar la interacción entre los factores involucrados que más afectan la determinación de la viscosidad dentro del proceso de evaporación-cristalización de soluciones azucaradas; tales como la concentración (en grados Brix, ${ }^{\circ} \mathrm{Bx}$ ), la temperatura y las revoluciones por minuto, $\mathrm{rpm}$ (velocidades de corte). En cada factor se consideraron tres niveles, por lo que se tiene un total de 27 experimentos a realizar, con su respectiva réplica (Montgomery, 2001). El orden corresponde al que se muestra en la Tabla 2.

Tabla 2 Factores y niveles utilizados en el diseño de experimentos.

\begin{tabular}{|c|c|c|c|c|c|c|c|c|c|}
\hline \multirow{4}{*}{$\begin{array}{c}\text { CONCENTRACION } \\
\left({ }^{\circ} \mathrm{Bx}\right)\end{array}$} & \multicolumn{9}{|c|}{ Temperatura $\left({ }^{\circ} \mathrm{C}\right)$} \\
\hline & \multicolumn{3}{|c|}{25} & \multicolumn{3}{|c|}{30} & \multicolumn{3}{|c|}{40} \\
\hline & \multicolumn{9}{|c|}{$\operatorname{RPM}\left(\mathrm{s}^{-1}\right)$} \\
\hline & 50 & 60 & 100 & 50 & 60 & 100 & 50 & 60 & 100 \\
\hline 45 & $1 / 28$ & $2 / 29$ & $3 / 30$ & $4 / 31$ & $5 / 32$ & $6 / 33$ & $7 / 34$ & $8 / 35$ & $9 / 36$ \\
\hline 60 & $10 / 37$ & $11 / 38$ & $12 / 39$ & $13 / 40$ & $14 / 41$ & $15 / 42$ & $16 / 43$ & $317 / 44$ & $18 / 45$ \\
\hline 70 & $19 / 46$ & $20 / 47$ & $21 / 48$ & $22 / 49$ & $23 / 50$ & $24 / 51$ & $25 / 52$ & 2 26/53 & $27 / 54$ \\
\hline
\end{tabular}

Este diseño se considera como completo debido al número de niveles y variables involucradas, sin embargo tiene una desventaja, el número de experimentos es elevado (27) y considerando la réplica mínima necesaria, debido a la sensibilidad de la variable de respuesta (viscosidad de la solución) con respecto a las variables del proceso, se requiere la realización de 54 corridas experimentales.

\section{Preparación de la solución azucarada}

La sustancia de experimentación es azúcar estándar de la zafra 2008-2009 con las características que se muestran en la Tabla 3, de acuerdo a las normas méxicanas.

Los experimentos se realizan empleando una solución inicial de $45^{\circ} \mathrm{Bx}$, con la finalidad de lograr una similitud con las condiciones de operación en los evaporadores de las fábricas de azúcar en un momento determinado, y posteriormente pasar a la etapa de cristalización. 
Esta solución se debe preparar para cada corrida, la cantidad requerida es de, aproximadamente, $500 \mathrm{ml}$ de solución, de acuerdo a la pasos siguientes. Para determinar la cantidad de azúcar requerida se toma en cuenta la definición de grados Brix.

${ }^{\circ} B x=100 \times \frac{\text { masa del soluto }}{\text { masa de la solución }}$

Tabla 3 Características fisicoquímicas del producto terminado. Ingenio La Margarita S.A. de C.V., zafra 2008-2009 (datos obtenidos del Ingenio La Margarita).

\begin{tabular}{cc}
\hline Variable & Valor \\
\hline Polarización & $99.40 \%$ \\
Humedad & $0.060 \%$ \\
Cenizas & $0.250 \%$ \\
Reductores & $0.100 \%$ \\
Color & Marfil o claro \\
Sabor & obscuro \\
& Dulce \\
\hline
\end{tabular}

Medición de la concentración en grados Brix

Cerciorarse de que el refractómetro esté a $0{ }^{\circ} \mathrm{Bx}$, para ello se debe colocar una gota de agua destilada y verificar la lectura inicial de $0{ }^{\circ} \mathrm{Bx}$. Poner una gota de la solución problema en el prisma del refractómetro y medir su concentración en ${ }^{\circ} \mathrm{Bx}$. Se procede de la misma forma con otras soluciones.

\section{Determinación de la viscosidad a diferentes temperaturas}

La metodología siguiente permite determinar la viscosidad a diferentes temperaturas: 1) Se agrega agua al rotavapor hasta un cuarto de su capacidad. 2) Se conecta el rotavapor a la corriente eléctrica. 3) Observar los incrementos de la temperatura del agua. 4) Como la prueba de medición de viscosidad durará $120 \mathrm{~min}$., aproximadamente, se recomienda medir continuamente la temperatura tanto del agua del rotavapor como la de la muestra cada $5 \mathrm{~min}$, y así establecer parámetros de operación, para tener un mayor control de la temperatura al momento de realizar las corridas experimentales con los tres diferentes valores de temperatura.

La metodología que se propuso, se fundamenta en la realización de un diseño de experimentos de tipo factorial $3^{\mathrm{k}}$, en donde se considera la interacción de tres factores en la determinación de la variable de respuesta, viscosidad de la solución.

\section{RESULTADOS Y DISCUSIÓN}

Se desarrollaron 27 experimentos con una réplica, haciendo un total de 54 experimentos realizados en forma aleatoria; los datos experimentales fueron capturados en el programa estadístico NCSS 97 R 1999 para el análisis de varianza, el cual proporciona una media de los datos obtenidos en el diseño de experimentos cuando intervienen los tres factores, estos se presentan en la Tabla 4.

Tabla 4 Media de la interacción de los tres factores en el diseño de experimentos.

\begin{tabular}{|c|c|c|c|c|c|c|c|c|c|}
\hline \multirow{4}{*}{$\begin{array}{c}\text { CONCENTRACIÓN } \\
\left({ }^{\circ} \mathrm{Bx}\right)\end{array}$} & \multicolumn{9}{|c|}{ TEMPERATURAS $\left({ }^{\circ} \mathrm{C}\right)$} \\
\hline & \multicolumn{3}{|c|}{25} & \multicolumn{3}{|c|}{30} & \multicolumn{3}{|c|}{40} \\
\hline & \multicolumn{9}{|c|}{$\operatorname{RPM}\left(s^{-1}\right)$} \\
\hline & 50 & 60 & 100 & 50 & 60 & 100 & 50 & 60 & 100 \\
\hline 45 & 25.85 & 29.65 & 33.75 & 24.00 & 26.75 & 32.05 & 21.4 & 22.20 & 27.60 \\
\hline 60 & 80.60 & 88.50 & 128.40 & 59.45 & 70.95 & 77.80 & 49.70 & 50.70 & 60.25 \\
\hline 70 & 320.80 & 326.35 & 344.55 & 198.90 & 209.75 & 225.20 & 114.5 & 120.45 & 168.8. \\
\hline
\end{tabular}

Para observar el comportamiento reológico que presentan las soluciones azucaradas se realizó un análisis estadístico de los datos de viscosidad de las soluciones. En esta sección se presentan los resultados experimentales así como también los valores numéricos que se obtuvieron a partir de los modelos estadísticos propuestos. Estos resultados se condensan en tablas y figuras con sus respectivas observaciones y comentarios de acuerdo a los diferentes niveles de experimentación.

Para calcular la velocidad de corte, esfuerzo cortante y viscosidad para ejes cilíndricos se utilizaron las ecuaciones: 
Velocidad de corte:

$$
S=\frac{2 \omega R_{c}^{2} R_{b}^{2}}{x^{2}\left(R_{c}^{2}-R_{b}^{2}\right)}
$$

Esfuerzo de corte:

$$
F^{\prime}=\frac{M}{2 \pi R_{b}^{2} L}
$$

Viscosidad:

$$
\eta=\frac{F}{S}
$$

donde $\omega$ es la velocidad angular del eje, $R_{c}$ es el radio del contenedor, $R_{b}$ es el radio del eje, $x$ es el radio en el que la velocidad de corte se calcula y $L$ es la longitud del eje.

\section{Viscosidades a una temperatura de $25^{\circ} \mathrm{C}$}

En la Tabla 5, se muestran los datos experimentales que se obtuvieron para un nivel de temperatura de $25{ }^{\circ} \mathrm{C}$. El esfuerzo de corte y la velocidad de corte se calcularon de acuerdo a las Ecuaciones (3) y (4). En el caso de la viscosidad, se realizó una estimación siguiendo un modelo de promedios a partir de los valores obtenidos en las corridas experimentales con sus respectivas réplicas.

Tabla 5 Características reológicas a $25^{\circ} \mathrm{C}$.

\begin{tabular}{ccccccc}
\hline $\begin{array}{c}\text { Concentración } \\
\left({ }^{\circ} \mathbf{B x}\right)\end{array}$ & eje & RPM & $\begin{array}{c}\text { Lectura } \\
\text { del } \\
\text { Viscosímetro }\end{array}$ & $\begin{array}{c}\text { Viscosidad } \\
{[1]}\end{array}$ & $\begin{array}{c}\text { Esfuerzo } \\
\text { Cortante } \\
\left(\text { dina/cm }^{2}\right)[2]\end{array}$ & $\begin{array}{c}\text { Velocidad } \\
\text { de Corte } \\
\text { s- }^{-1}\end{array}$ \\
\hline $\mathbf{4 5}$ & 1 & 50 & 26.75 & 53.5 & 1.45 & 2.72 \\
& 1 & 60 & 28.05 & 46.75 & 1.83 & 3.91 \\
& 1 & 100 & 34.35 & 34.35 & 3.73 & 10.87 \\
60 & 1 & 50 & 65.65 & 131.3 & 3.5 & 2.72 \\
& 1 & 60 & 70 & 116.67 & 4.5 & 3.91 \\
& 1 & 100 & 90.15 & 90.15 & 9.8 & 10.87 \\
70 & 2 & 50 & 276.1 & 2208.8 & 5.39 & 0.24 \\
& 2 & 60 & 278.55 & 1857 & 6.53 & 0.35 \\
& 2 & 100 & 300.9 & 1203.6 & 11.75 & 0.98 \\
\hline
\end{tabular}

[1] Para el cálculo de este valor se multiplicó la lectura de viscosidad en cP por su Factor Eje/Velocidad (Brookfield, 2003)

[2] Para el cálculo de este valor se utilizo la Ecuación (4).

En las Figs. 4 y 5, se muestran los reogramas construidos a partir de los datos de la Tabla 5, con la finalidad de observar el comportamiento reológico de las soluciones a $25^{\circ} \mathrm{C}$.

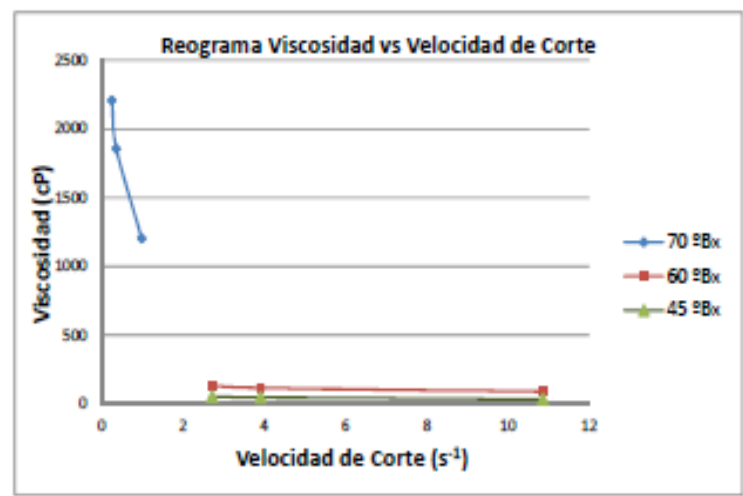

Fig. 4 Comportamiento reológico a $25^{\circ} \mathrm{C}$.

Se puede apreciar que el comportamiento de las soluciones azucaradas de trabajo, se asemeja al de un fluido pseudoplástico, es decir, al aumentar la velocidad de corte la viscosidad disminuye, considerando los tres niveles de concentración en estudio, Fig. 4. Sin embargo, la viscosidad de la solución a $70{ }^{\circ} \mathrm{Bx}$ presenta un cambio drástico con respecto a las otras concentraciones, debido a que a esa concentración empiezan a formarse cristales en la solución, además de que el tamaño del eje (No. 2) utilizado para la medición de la viscosidad dió una concentración diferente al eje utilizado para medir la viscosidad de las otras soluciones (No. 1). Este mismo fenómeno se observa en la Fig. 5, en donde se gráfica el comportamiento del esfuerzo cortante respecto a la velocidad de corte.

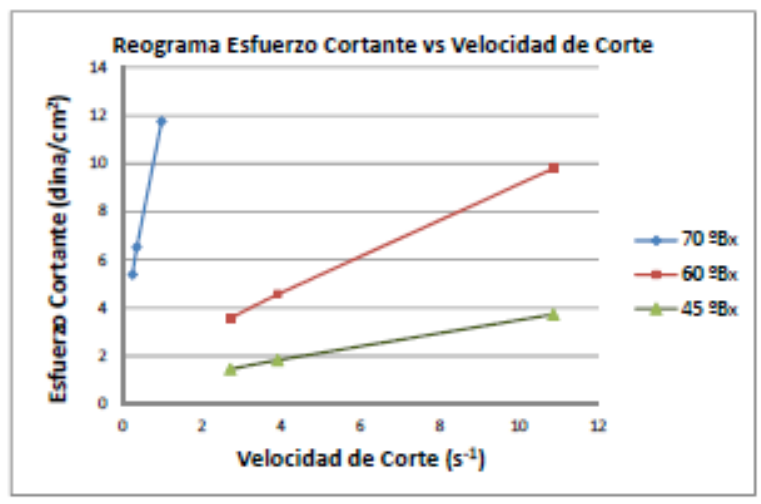

Fig. 5 Reograma esfuerzo cortante vs velocidad de corte a $25^{\circ} \mathrm{C}$. 
Viscosidades a una temperatura de $30^{\circ} \mathrm{C}$

De igual manera con los datos de la Tabla 6, se obtuvo un promedio de datos del experimento y su respectiva réplica a una temperatura de $30^{\circ} \mathrm{C}$. En la Tabla 5, se muestran los resultados experimentales para un nivel de temperatura de $30{ }^{\circ} \mathrm{C}$, los valores se calcularon con las ecuaciones (3) y (4) para el esfuerzo cortante y la velocidad de corte. De igual manera que en el nivel de temperatura de $25{ }^{\circ} \mathrm{C}$, la viscosidad se calculó, con el modelo promediado descrito en la Ecuación (5).

Tabla 6 Características reológicas a $30^{\circ} \mathrm{C}$

\begin{tabular}{ccccccc}
\hline $\begin{array}{c}\text { Concentración } \\
\left({ }^{\circ} \mathrm{Bx}\right)\end{array}$ & Eje & RPM & $\begin{array}{c}\text { Lectura } \\
\text { del } \\
\text { Viscosímetro }\end{array}$ & $\begin{array}{c}\text { Viscosidad } \\
(\mathrm{cP})[1]\end{array}$ & $\begin{array}{c}\text { Esfuerzo } \\
\text { Cortante } \\
\left.\left(\text { dina/cm }^{2}\right)\right)[2]\end{array}$ & $\begin{array}{c}\text { Velocidad } \\
\text { de Corte } \\
\text { s- }^{-1}\end{array}$ \\
\hline $\mathbf{4 5}$ & 1 & 50 & 23.3 & 57.7 & 1.57 & 2.72 \\
& 1 & 60 & 28.85 & 38.83 & 1.52 & 3.91 \\
& 1 & 100 & 31.05 & 31.05 & 3.37 & 10.87 \\
60 & 1 & 50 & 50.95 & 101.9 & 2.77 & 2.72 \\
& 1 & 60 & 58.2 & 97 & 3.79 & 3.91 \\
& 1 & 100 & 68 & 68 & 7.39 & 10.87 \\
70 & 2 & 50 & 184.85 & 1478.8 & 3.61 & 0.24 \\
& 2 & 60 & 201.05 & 1340.3 & 4.71 & 0.35 \\
& 2 & 100 & 227.25 & 909 & 8.87 & 0.98 \\
\hline
\end{tabular}

En las Figs. 6 y 7, se muestran los reogramas realizados a partir de los datos de la Tabla 5, aquí se observa el comportamiento reológico de las soluciones a $30^{\circ} \mathrm{C}$.

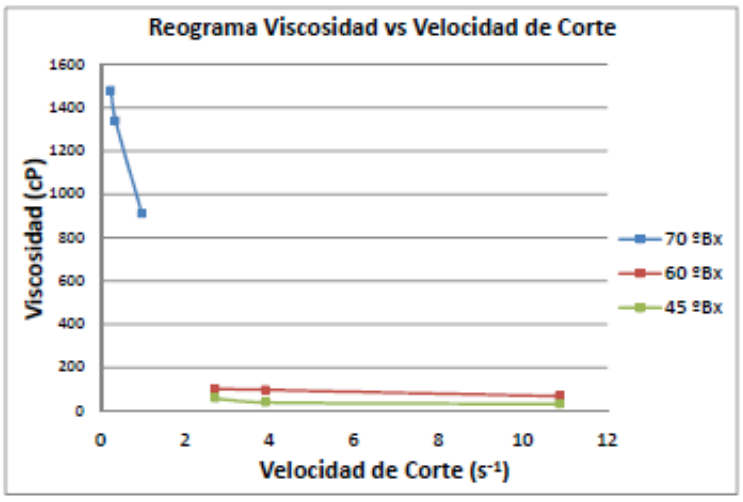

Fig. 6 Comportamiento reológico a $30^{\circ} \mathrm{C}$.

En la Fig. 6, se observa el comportamiento de las soluciones azucaradas similar al de un fluido pseudoplástico, debido a que al aumentar la velocidad de corte la viscosidad disminuye. La viscosidad de la solución a $70{ }^{\circ} \mathrm{Bx}$, de igual manera que en el nivel de temperatura anterior, presenta un comportamiento con cambios más drásticos, respecto a los otros niveles de concentración de las soluciones $\left(45\right.$ y $\left.60{ }^{\circ} \mathrm{Bx}\right)$. Este fenómeno también se observa en la Fig. 7, con el reograma de esfuerzo cortante - velocidad de corte.

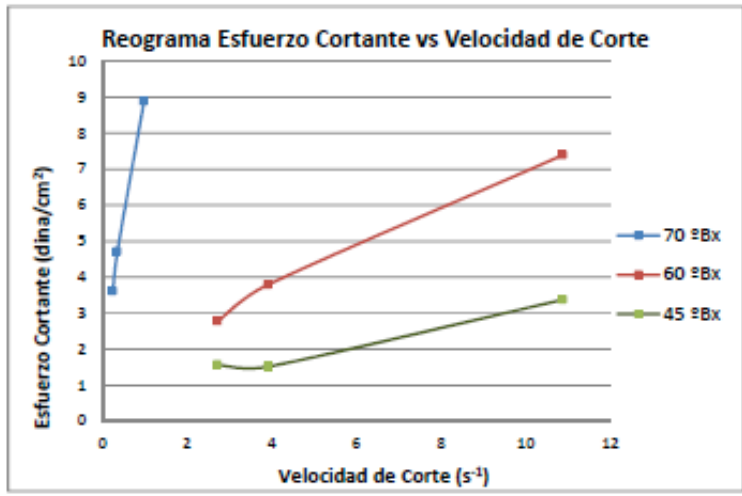

Fig. 7 Reograma esfuerzo cortante vs velocidad de corte a $30{ }^{\circ} \mathrm{C}$.

Viscosidades a una temperatura de $40{ }^{\circ} \mathrm{C}$

Para el nivel de temperatura de $40{ }^{\circ} \mathrm{C}$, se obtuvieron los datos que se muestran en la Tabla 7 la viscosidad calculada se obtuvo con el modelo promediado a partir de los resultados experimentales. El esfuerzo de corte y la velocidad se corte se calcularon con las Ecuaciones (3) y (4).

Tabla 7 Características reológicas a $40{ }^{\circ} \mathrm{C}$.

\begin{tabular}{ccccccc}
\hline $\begin{array}{c}\text { Concentración } \\
\left({ }^{\circ} \mathrm{Bx}\right)\end{array}$ & Eje & RPM & $\begin{array}{c}\text { Lectura } \\
\text { del } \\
\text { Viscosimetro }\end{array}$ & $\begin{array}{c}\text { Viscosidad } \\
(\mathrm{cP})\end{array}$ & $\begin{array}{c}\text { Esfuerzo } \\
\text { Cortante } \\
\left(\mathrm{dina} / \mathrm{cm}^{2}\right)\end{array}$ & $\begin{array}{c}\text { Velocidad } \\
\text { de Corte } \\
\text { s }^{-1}\end{array}$ \\
\hline $\mathbf{4 5}$ & 1 & 50 & 20.85 & 41.7 & 1.13 & 2.72 \\
& 1 & 60 & 22.15 & 36.92 & 1.44 & 3.91 \\
& 1 & 100 & 25.85 & 25.85 & 2.81 & 10.87 \\
$\mathbf{6 0}$ & 1 & 50 & 39.15 & 78.38 & 2.81 & 2.72 \\
& 1 & 60 & 46 & 76.67 & 3.0 & 3.91 \\
& 1 & 100 & 59.95 & 59.95 & 6.52 & 10.87 \\
70 & 1 & 50 & 97.9 & 195.8 & 5.32 & 2.72 \\
& 1 & 60 & 105.75 & 176.25 & 6.89 & 3.91 \\
& 1 & 100 & 98.8 & 98.5 & 10.71 & 10.87 \\
\hline
\end{tabular}

En las Figs. 8 y 9 se muestran los reogramas realizados con los datos de la Tabla 6 , en donde se observa el comportamiento reológico presentado por las soluciones es semejante al de un fluido pseudoplástico. 
Es necesario mencionar que a este nivel de temperatura se utilizó un mismo eje (No. 1) para medir la viscosidad de las tres soluciones; por lo tanto no se observan comportamientos irregulares o drásticos en las mediciones a diferencia de las que se realizaron en los niveles de $30{ }^{\circ} \mathrm{C}$ y $25{ }^{\circ} \mathrm{C}$. El comportamiento es semejante cuando se observa el esfuerzo de corte con la velocidad de corte, Fig. 9.

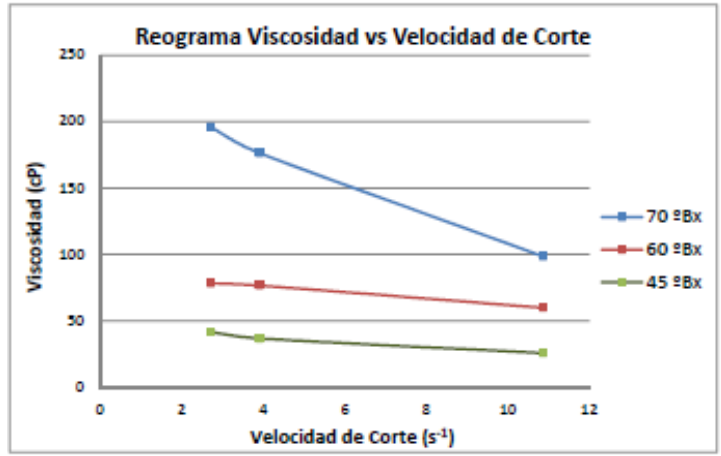

Fig. 8 Comportamiento reológico a $40{ }^{\circ} \mathrm{C}$.

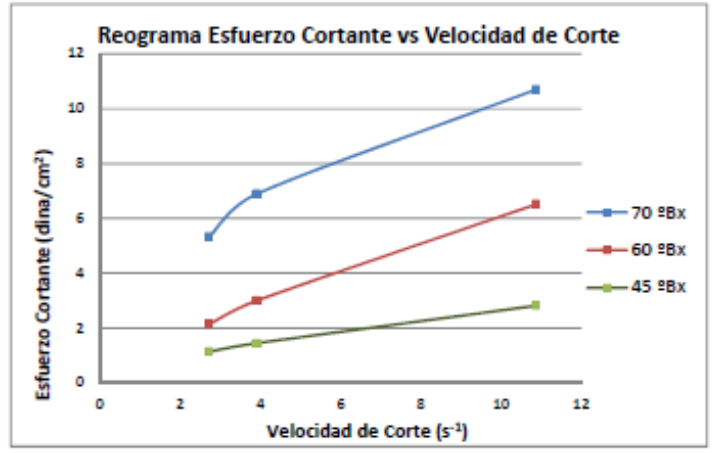

Fig. 9 Reograma esfuerzo cortante vs velocidad de corte a $40{ }^{\circ} \mathrm{C}$.

En las Figs. 10 y 11, se puede observar el comportamiento dilatante que presentan las soluciones azucaradas a una temperatura de 40 ${ }^{\circ} \mathrm{C}$.

\section{Discusión de resultados}

Para construir el modelo matemático que describe el comportamiento reológico observado de las soluciones de trabajo, se hicieron correlaciones y análisis de los datos obtenidos en las corridas experimentales en el programa estadístico NCSS 97 R 1999. Se aplicó la función "multivariable ratio of polynomials report", que proporciona un modelo simbólico y un modelo estimado.

Debido al comportamiento que se observa en los datos experimentales a concentraciones altas (70 $\left.{ }^{\circ} \mathrm{Bx}\right)$, se propuso obtener un modelo para el rango de concentraciones de bajas y medias (45 a $60^{\circ} \mathrm{Bx}$ ) y otro para concentraciones altas.

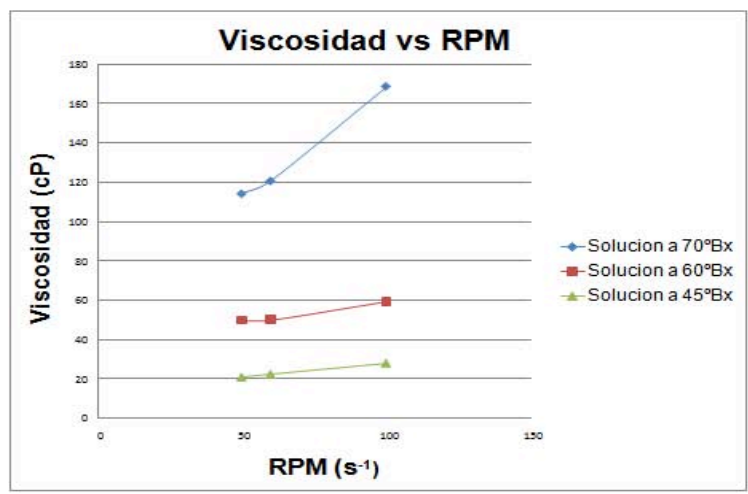

Fig. 10 Reograma viscosidad vs. rpm a $40{ }^{\circ} \mathrm{C}$.

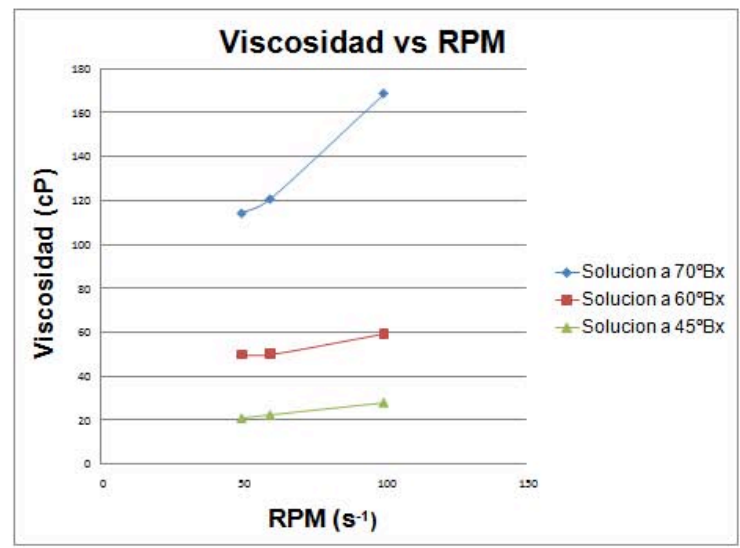

Fig. 11 Reograma esfuerzo cortante vs. rpm a $40{ }^{\circ} \mathrm{C}$.

En la Ecuación (6), se observa el modelo proporcionado por el programa estadístico, para concentraciones de 45 y $60{ }^{\circ} \mathrm{Bx}$, con un factor de correlación de 0.886134 . 


$$
\begin{gathered}
\mu=57.12027-0.82255699 T+\ldots \\
6.074852 E^{-8}\left(T^{\circ} B x r p m \mu^{2}\right)
\end{gathered}
$$

De la misma forma se obtuvo un modelo para 70 ${ }^{\circ} \mathrm{Bx}$ con un factor de correlación de 0.881028 , ver Ecuación (7).

$$
\begin{gathered}
\mu=8305.38-197.0545(T)-\ldots \\
5.326205 E^{-8}\left(\text { Trpm }^{2}\right)
\end{gathered}
$$

donde ${ }^{\circ} \mathrm{Bx}$, es la concentración en grados Brix, $T$ es la temperatura, $\mu$ es la viscosidad y rpm son revoluciones por minuto.

En la Tabla 8, se muestra un análisis comparativo entre la viscosidad obtenida en forma experimental y la viscosidad calculada con el modelo estadístico obtenido con el software NCSS. Se muestran los errores relativos que se alcanzan para diferentes condiciones de trabajo, a una sola velocidad de corte, de los tres niveles de velocidad estudiados.

Tabla 8 Error relativo de viscosidades experimentales y calculadas.

\begin{tabular}{cccccc}
\hline $\begin{array}{c}\text { Temp } \\
\left({ }^{\circ} \mathrm{C}\right)\end{array}$ & $\begin{array}{c}\text { Conc } \\
\left({ }^{\circ} \mathrm{Bx}\right)\end{array}$ & $\begin{array}{c}\text { Velocidad } \\
\text { de corte }\end{array}$ & $\begin{array}{c}\text { Viscosidad } \\
\text { experimental }\end{array}$ & $\begin{array}{c}\text { Viscosidad } \\
\text { NCSS }\end{array}$ & $\begin{array}{c}\text { Error } \\
\text { relativo }\end{array}$ \\
\hline \multirow{2}{*}{25} & 45 & 3.9194123 & 46.71 & 45.52 & 0.02547634 \\
& 60 & 3.9194123 & 116.66 & 110.98 & 0.04868849 \\
& 70 & 3.9194123 & 1857 & 1895.33 & -0.0206408 \\
& 45 & 3.9194123 & 38.83 & 39.86 & -0.0265258 \\
30 & 60 & 3.9194123 & 97 & 94.18 & 0.02907216 \\
& 70 & 3.9194123 & 1340.33 & 1108.68 & 0.17283242 \\
& 45 & 3.9194123 & 36.92 & 33.16 & 0.10184182 \\
40 & 60 & 3.9194123 & 76.66 & 75.62 & 0.01356639 \\
& 70 & 3.9194123 & 176.25 & 197.83 & -0.1224397 \\
\hline
\end{tabular}

De acuerdo a los datos que se presentan en la Tabla 8 , se observan errores relativos máximos de hasta $17 \%$, por lo que se puede afirmar que los modelos obtenidos son aceptables para cálculos preliminares en análisis y simulación de operaciones en donde se tienen condiciones similares a las de los experimentos desarrollados.

El desarrollo experimental y el análisis de resultados condujo a la determinación del modelo reológico de las soluciones problema en el rango de estudio de los factores de interés, proponiéndose un modelo tipo Ostwald- de Waele, conocido como ley de potencia, para fluidos dilatantes (Bird et al., 2002).

También, a partir de los resultados experimentales se desarrollaron análisis estadísticos y numéricos que correlacionan a la viscosidad con la temperatura y la concentración, principalmente, esto se muestra en la Ecuación (8).

En la Fig. 12, se trazaron los datos de viscosidad correspondientes a las tres concentraciones de trabajo $\left(45,60\right.$ y $\left.70{ }^{\circ} \mathrm{Bx}\right)$ en función de la temperatura $\left(25,30\right.$ y $\left.40^{\circ} \mathrm{C}\right)$.

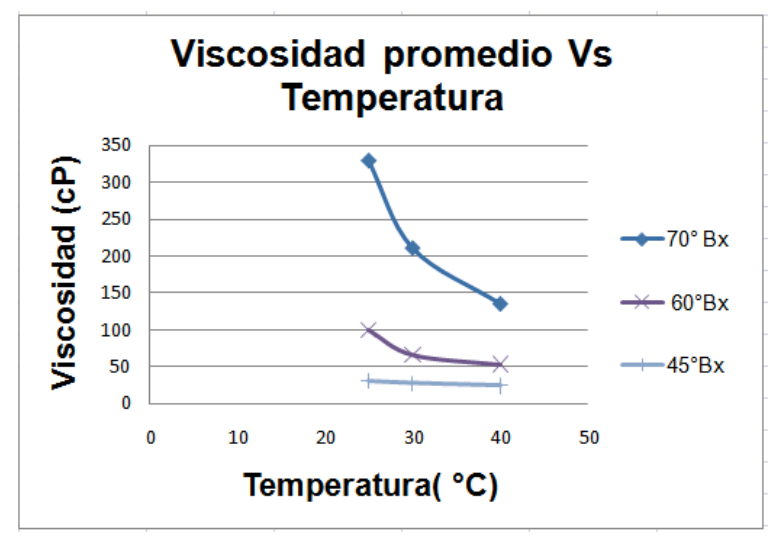

Fig. 12 Viscosidad en función de la temperatura a diferentes concentraciones.

Se puede observar en la Tabla 9, los resultados que se obtienen del modelo de correlación y la comparación entre el valor experimental promedio de la viscosidad y el valor calculado con el modelo.

$\mu\left(T,{ }^{\circ} B x\right)=a\left({ }^{\circ} B x\right) x^{2}+b\left({ }^{\circ} B x\right) x+c\left({ }^{\circ} B x\right)$

donde

$$
\begin{aligned}
& a\left({ }^{\circ} \mathrm{B} x\right)=0.002\left({ }^{\circ} \mathrm{Bx}\right)^{2}-0.1915\left({ }^{\circ} \mathrm{B} x\right)+4.5082 \\
& b\left({ }^{\circ} \mathrm{B} x\right)=0.1653\left({ }^{\circ} \mathrm{Bx}\right)^{2}-15.72\left({ }^{\circ} \mathrm{B} x\right)+8360.3 \\
& c\left({ }^{\circ} \mathrm{Bx}\right)=3.5988\left({ }^{\circ} \mathrm{B} x\right)^{2}-346.47\left({ }^{\circ} \mathrm{B} x\right)+8360.3
\end{aligned}
$$


Tabla 9 Resultados numéricos y comparación entre valores experimentales y calculados.

\begin{tabular}{cccccc}
\hline $\begin{array}{c}\text { Tem } \\
\left({ }^{\circ} \mathbf{C}\right)\end{array}$ & $\begin{array}{c}\text { Conc } \\
\left({ }^{\circ} \mathbf{B x}\right)\end{array}$ & $\begin{array}{c}\text { Viscosidad } \\
\text { Experim }\end{array}$ & $\begin{array}{c}\text { Viscosidad } \\
\text { Promedio }\end{array}$ & $\begin{array}{c}\text { Viscosidad } \\
\text { Calculada }\end{array}$ & $\begin{array}{c}\text { Error } \\
\text { Relativo }\end{array}$ \\
\hline \multirow{4}{*}{25} & 70 & 886.77 & & & \\
& 60 & 687.55 & 743.555 & 743.57 & 0.002689781 \\
& 45 & 656.34 & & & \\
& 70 & 851.3 & & & \\
30 & 60 & 751.76 & 790.145 & 790.17 & 0.003163976 \\
& 45 & 767.37 & & & \\
& 70 & 915.84 & & & \\
40 & 60 & 913.7 & 936.693 & 936.73 & 0.003914479 \\
& 45 & 980.54 & & & \\
\hline
\end{tabular}

En forma adicional, se presentan los resultados numéricos de la Fig. 13, en donde se observa el comportamiento de la viscosidad promedio en función de la temperatura, como una variable importante que influye en la caracterización de soluciones azúcaradas.

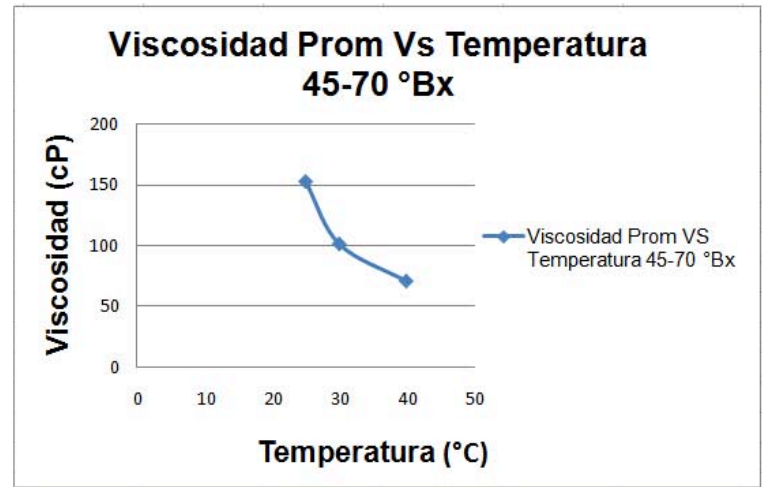

Fig. 13 Viscosidad promedio contra temperatura.

En resumen, con base en la solución de trabajo se construyó una metodología para la caracterización reológica de la solución azucarada; aplicable a etapas del proceso de producción de azúcar, principalmente, en las etapas de evaporación y cristalización. Debido a las limitantes en cuanto a la materia prima en la fábrica de azúcar por la naturaleza estacional del proceso de producción (noviembre-mayo), se espera que en próximos trabajos se aplique esta metodología directamente en meladuras (evaporación) y masa cocida (cristalización). Igualmente, se plantea el desarrollo de pruebas experimentales a las temperaturas de trabajo en el proceso real para la construcción de perfiles reológicos, a realizarse en una etapa posterior.

El desarrollo del trabajo ha permitido poner a punto la metodología para realizar esta caracterización, con opción de extender este trabajo a un rango mayor de concentración y temperatura para equiparar las condiciones de operación en los equipos industriales del proceso de producción de azúcar. Además, en forma paralela a este trabajo se tienen algunos resultados sobre la caracterización de mieles finales y productos intermedios en términos de pureza, para disminuir pérdidas de sacarosa en la industria azucarera.

\section{CONCLUSIONES}

La caracterización reológica de soluciones azucaradas para el proceso de evaporación cristalización a concentraciones de 45, 60 y 70 ${ }^{\circ} \mathrm{Bx}$, a diferentes temperaturas $\left(25,30\right.$ y $\left.40{ }^{\circ} \mathrm{C}\right) \mathrm{y}$ velocidades de corte (50, 60 y $100 \mathrm{rpm})$, permitió la elaboración de perfiles reológicos de las soluciones que presentan un comportamiento semejante al de los fluidos que siguen la ley de la potencia de Ostwald-de Waele.

A través del planteamiento factorial del diseño de experimentos se identificó que las variables que más afectan a la variable de respuesta es la concentración y la temperatura y en menor proporción las rpm.

El error relativo que se observó tiene una magnitud máxima de $17 \%$, de acuerdo a la metodología experimental propuesta y a la secuencia de análisis de correlaciones realizada, con base en estos resultados se afirma que el modelo obtenido es aceptable para cálculos preliminares en análisis y simulación de procesos en condiciones similares a los experimentos realizados. La obtención del modelo de viscosidad que correlaciona la concentración en ${ }^{\circ}$ Brix y la temperatura, a pesar de que el rango de validez experimental está en un margen limitado, muestra la metodologia a seguir para construir un mapa de interacción de estas variables en los procesos de evaporación y cristalización de azucar de caña. 


\section{AGRADECIMIENTOS}

Un agradecimiento especial al FOMIX CONACYT-Veracruz por el apoyo para la realización y presentación de este trabajo, Proyecto 128573. Los autores también desean agradecer a Martín García García por el análisis estadístico realizado y a Sarahi Pacheco Espinoza y Mayra Luna Trujillo por el desarrollo experimental, para la integración final de este trabajo.

\section{NOTACIÓN}

\begin{tabular}{|c|c|c|}
\hline$F$ & Esfuerzo de corte & $\left(\mathrm{dyn} / \mathrm{cm}^{2}\right)$ \\
\hline$K$ & Constante en Ecuación (1) & $(\mathrm{cP})$ \\
\hline$L$ & Longitud del eje & $(\mathrm{cm})$ \\
\hline$M$ & Fuerza aplicada & (dyn) \\
\hline$n$ & Constante en Ecuación (1) & $(-)$ \\
\hline$R_{b}$ & Radio del eje & $(\mathrm{cm})$ \\
\hline$R_{c}$ & Radio del contenedor & $(\mathrm{cm})$ \\
\hline$S$ & Velocidad de corte & $(1 / \mathrm{s})$ \\
\hline$T$ & Temperatura & $\left({ }^{\circ} \mathrm{C}\right)$ \\
\hline$x$ & $\begin{array}{l}\text { Radio en el que la velocidad } \\
\text { de corte se calcula }\end{array}$ & $(\mathrm{cm})$ \\
\hline \multicolumn{3}{|c|}{ Letras griegas } \\
\hline$\hat{\gamma}$ & Velocidad de deformación & $(1 / \mathrm{s})$ \\
\hline$\eta$ & Viscosidad aparente & (cP) \\
\hline$\mu$ & Viscosidad dinámica & (cP) \\
\hline$\tau$ & Esfuerzo cortante & $(\mathrm{m} \cdot \mathrm{Pa})$ \\
\hline$\omega$ & Velocidad angular & $(\mathrm{rad} / \mathrm{s})$ \\
\hline
\end{tabular}

\section{REFERENCIAS}

Bird, R.B., Steward, W.E. \& Lightfoot, E.N. (2002). Transport Phenomena, 2nd Ed. New York, USA: John Wiley \& Sons, Inc.

Brookfield Engineering Laboratories, Inc. (2003). More Solutions to Sticky Problems. USA.

Chen, J.C.P \& Chen, M. (2000). Manual del Azúcar de Caña. México D.F., México: Editorial Limusa.

Díaz García, A.A. \& Remedios Castañeda, P.D. (2002). Análisis de las viscosidades de mieles y masas cocidas de las provincias de Santiago de
Cuba y Las Tunas con vistas a establecer las variaciones más esperadas con la temperatura. Tecnología Química, Vol. XXII, No. 2, pp. 4958. ISSN 0041-8420.

Montgomery, D.C. (2001). Design and Analysis of Experiments, 6th Ed. New York, USA: John Wiley \& Sons, Inc.

Shames, I.H. (1999). Mecánica de Fluidos, 3ra Ed. Colombia: McGraw-Hill.

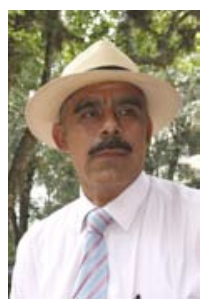

Anselmo Osorio Mirón es Doctor en Ciencias en la especialidad de Control Automático del CINVESTAVIPN, México. Profesor de Tiempo Completo, Titular $\mathrm{C}$ en la Facultad de Ciencias Químicas, Universidad Veracruzana, México. Sus áreas de intereses son: control y simulación de procesos químicos; sistemas no lineales; observadores de estado; detección de fallas; análisis, modelado y simulación de procesos químicos y biológicos; matemáticas aplicadas; dinámica de fluidos computacional y aplicaciones. 